\title{
Stereological Analysis of the Vascular Network in Superior Colliculi
}

\author{
Igor Sladojević, ${ }^{1}$ Teodora Prerad, ${ }^{1}$ Mirjana Mršić ${ }^{1}$
}

\begin{abstract}
Background/Aim: Superior colliculi have an important role in the visual system, some motor functions and behaviour. Branches of the posterior cerebral artery and surrounding vessels form a collicular arterial network, which gives rise to several penetrating vessels for superior colliculi. The aim of the study was to determine relative stereological parameters (volume, surface and length density) of the vascular network in superior colliculi and to correlate those parameters with age.

Methods: Ten adult brainstems, both male and female, were cut transversally at the middle of superior colliculi in three-millimetre-thick strata. Every stratum was cut in $4 \mu \mathrm{m}$ thick semiserial sections stained with Mallory method. Images of every fifth section $(5,10,15,20)$ of superior colliculus were taken with „Leica“ DM 1000 microscope and „Leica“ MC 170HD camera under x 400 magnification and analysed by ImageJ software with A 100 grid. Statistical analysis was performed by the SPSS software with $5 \%$ level of significance using methods of descriptive statistics, Spearman's and Pearson's correlation coefficient.

Results: The average volume density of vascular network in superior colliculi was $0.0203 \mathrm{~mm}^{0}$, surface density $6.133 \mathrm{~mm}^{-1}$, and length density $205.357 \mathrm{~mm}^{-2}$. All those parameters decreased with ageing, but statistically significant change was seen only in length density $(\mathrm{p}=0.006)$.

Conclusion: Compared to the structures at the same transversal level, the vascular network of superior colliculi has an average density. With ageing, blood vessels become less tortuous.
\end{abstract}

Key words: Superior colliculus; Blood vessels; Ageing.
(1) Department of Anatomy, Faculty of Medicine, University of Banja Luka, Banja Luka, the Republic of Srpska, Bosnia and Herzegovina.

Correspondence: IGOR SLADOJEVIĆ $\mathrm{T:}+38751234100$

E: igor.sladojevic@med.unibl.org

\section{ARTICLE INFO}

Received: 5 October 2020 Revision received: 10 November 2020 Accepted: 11 November 2020

\section{Introduction}

The superior colliculi (SC) are subcortical visual centres that control eye movements and process visual information. ${ }^{1} \mathrm{SC}$ also has a role in motor functions, which helps humans to orient their attention and behaviour according to sensitive information. ${ }^{2}$ Besides, SC generates voluntary and reflex saccades. ${ }^{3} \mathrm{SC}$ lesions are most commonly vascular and cause the paralysis of upward gaze, blurry vision, dizziness and sometimes even paraesthesias of the lateral part of the upper lip. ${ }^{4}$ Terminal branches of the collicular artery (P1 segment of the posterior cerebral artery) supply SC. If these branches have a smaller calibre, the superior cerebellar artery supplies the lower part of SC and the most rostral part receives blood from branches of the posterior medial choroidal artery., 6 Those branches form a collicular network on the SC surface, ${ }^{6}$ which gives rise to several thin branches that penetrate each SC, passing through all layers of SC as parallel arches, concave mediodorsally. There are two types of penetrating branches: short and long. Short ones reach the middle layers of SC, while long ones pass through all layers of SC and give branches to the periaqueductal grey 
matter. ${ }^{7}$ Distribution of blood vessels within SC is even and, based only on the blood vessels density, it is not possible to distinguish layers of SC nucleus. ${ }^{5}$ Those vessels have ipsilateral anastomoses, and vascular fields of left and right SC are separated by an avascular zone, which corresponds to vertical intercollicular sulcus..$^{5}$ Due to these facts, vascular lesions in SC are ipsilateral and in some cases there is no neurological defect. ${ }^{8,9}$

The aim of the study is to perform a stereological analysis of blood vessels of SC by determining the relative stereological parameters: volume, surface, and length density, and to determine possible changes in these parameters with ageing.

\section{Methods}

The study was conducted with the approval of the Ethics Committee of the University Clinical Centre of the Republic of Srpska on samples of 10 brainstems, both sexes, aged 21 to 83 years (average age 57.07 years), who died without diagnosis of neurological diseases. Brainstems were cut into two three-millimetre-thick strata in the transverse plane (stratified sampling) at the level of the middle of the SC. ${ }^{10}$ Semi-serial sections $(5,10,15$, 20), $4 \mu \mathrm{m}$ thick, were made from the obtained strata and stained with the Mallory method. The reference space of the study in all cases was SC, and the studied phase was the blood vessels.

Photographs of the research object were taken using a camera "Leica" MC 170HD (Leica Microsystems CMS GmbH, Wetzlar, Germany), TIFF format, resolution $2592 \times 1944$ pixels, at magnification x400 light microscope "Leica" DM 1000 (Leica Microsystems CMS GmbH, Wetzlar, Germany) and $\mathrm{x} 0.7$ magnification of the camera c-mount. A total of 6,000 visual fields were photographed. ImageJ, version 1.53a (National Institutes of Health, Bethesda, USA) was used for stereological analysis. Before the analysis, a spatial calibration was performed using an object micrometre and the parameters of the A 100 test system were determined, based on which, using the grid option, a test system A 100 was formed (Table 1).

After setting up the grid test system, the image was analysed using a cell counting tool. In each section, 10 visual fields were measured: scores, intersections, and the number of blood vessels, and the values obtained were included in formulas for calculating relative stereological parameters (volume, surface, and length density).

\section{Table 1: Basic parameters of the test system A 100}

\begin{tabular}{cc}
\hline A 100 & Objective $\mathbf{x}$ 40 \\
\hline Pt & 100 \\
\hline d & $0,0193 \mathrm{~mm}$ \\
\hdashline Lt & $3,86 \mathrm{~mm}$ \\
\hdashline At & $0,0374 \mathrm{~mm}^{2}$ \\
\hline
\end{tabular}

Pt - number of points in A 100 test system; $d$ - length of one line of the test system; $L t-$ length of all lines of the test system; At - the surface of the test system; $L t=P t \cdot d \cdot 2 ; A t=P t \cdot d^{2}$

Volume density (Vv) was calculated according to the formula: $\mathrm{Vv}\left(\mathrm{mm}^{0}\right)=$ Pf/Pt (Pf - number of points of the test system falling on the studied phase; $\mathrm{Pt}$ - total number of points within the test system A100). ${ }^{11}$

Surface density (Sv) was determined by the formula: $\mathrm{Sv}\left(\mathrm{mm}^{-1}\right)=2 \cdot$ If $/$ Lt (If- the number of intersections of test system lines with studies phase; Lt- total length of test lines). ${ }^{11}$

Length density (Lv) is a relative stereological parameter which determines the length of a certain curve in a volume unit. It was determined by the formula: $\mathrm{Lv}=2 \mathrm{x}$ Qf / At $\left(\mathrm{mm}^{-2}\right)$, where Qf represents the number of intersections of blood vessels and A 100 test system, and At is the surface of the test system. ${ }^{12}$

Statistical analysis of the results was performed using the Statistical Package for the Social Sciences (SPSS) software (SPSS Inc, Chicago, USA), version 16.0, using methods of descriptive statistics, Spearman's and Pearson's correlation coefficient. Statistical significance was tested for the level of statistical significance of $5 \%$.

\section{Results}

The study was conducted on 10 SCs, both sexes ( 5 men and 5 women). The youngest analysed sample was from a person aged 24 years, and the oldest 82 years (group average age 60.5 years).

The mean value of the volume density of blood vessels was $0.0203 \mathrm{~mm}^{0}$. The lowest volume density of blood vessels was in a person aged 66 years $\left(0.0151 \mathrm{~mm}^{0}\right)$ and the highest in a person aged 55 years $\left(0.0361 \mathrm{~mm}^{0}\right)$. The value of volume density decreased with age, but the decrease did 
not reach statistical significance $(\rho=-0.552 ; \mathrm{p}=$ 0.098) - Figure 1.

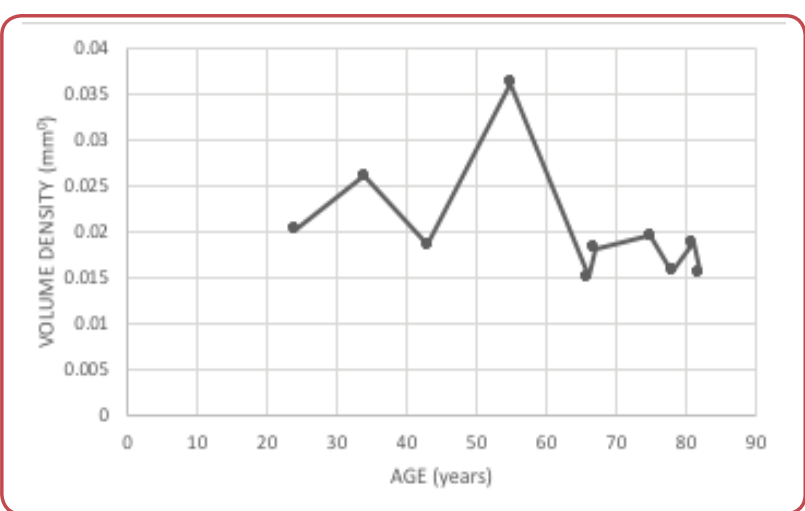

Figure 1: Values of volume density of blood vessels of the SC with age

The average value of the surface density of the blood vessels was $6.133 \mathrm{~mm}^{-1}$. The lowest surface density of blood vessels was in a person aged 78 years $\left(4.026 \mathrm{~mm}^{-1}\right)$ and the highest was found in a person aged 55 years $\left(9.335 \mathrm{~mm}^{-1}\right)$. Surface density values also decreased with age, but also without significance $(r=-0.49 ; p=0.151)$ - Figure 2 .

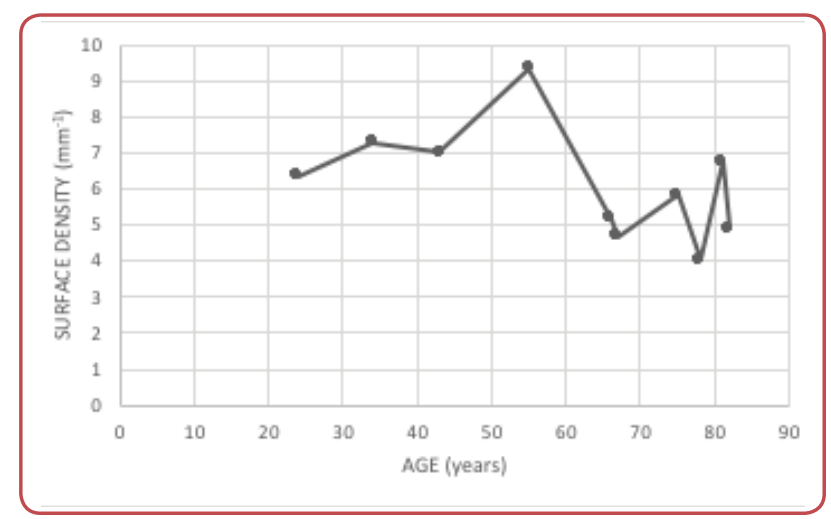

Figure 2: Surface density of SC with aging

The length density averaged $205.357 \mathrm{~mm}^{-2}$. The highest value was observed in a 34-year-old person $\left(295.882 \mathrm{~mm}^{-2}\right)$, and the lowest in an 82-year-

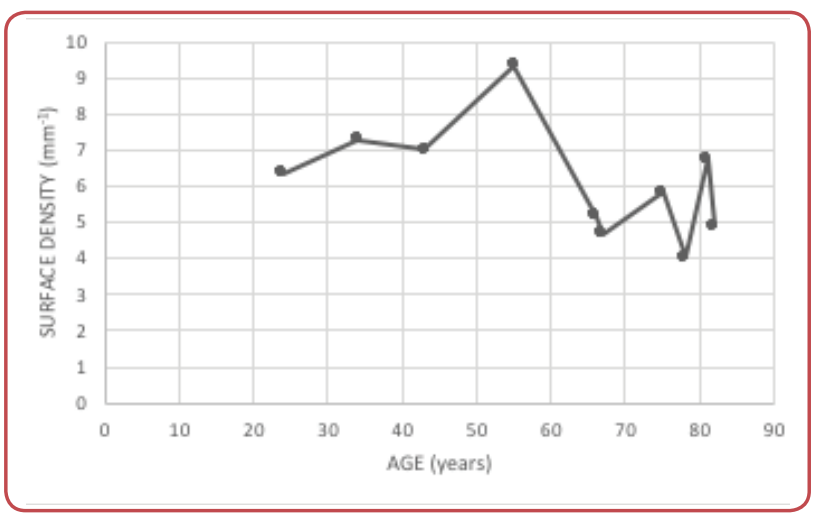

Figure 3: Changes of length density with aging old person (138.609 $\mathrm{mm}^{-2}$ ). In contrast to volume and surface densities, the decrease in length density with ageing was statistically significant $(\mathrm{r}=$ -0.799; $\mathrm{p}=0.006$ ) - Figure 3.

\section{Discussion}

Detailed knowledge of the size of the vascular fields within the midbrain is necessary to interpret the pathological changes that occur as a result of occlusion of the midbrain blood vessels. Available literature data describe pathological changes in terms of case reports, ${ }^{8}$ while data on stereological parameters of blood vessels of the SC and their eventual change with ageing are not found.

The midbrain arterial network is complex, which is why vascular lesions do not give symptoms of midbrain lesion only, but also symptoms of damage to the cerebellum, thalamus and pons. ${ }^{13}$ Clinical features of midbrain infarcts include a variety of symptoms, depending on size and location of the infarct. Dorsal midbrain infarct (where SC is located) causes oculomotor syndromes, diplopia and abnormalities of vertical gaze, but it can also include auditory symptoms, such as tinnitus and hyperacusis. ${ }^{14}$

The results obtained in this study were compared with the characteristics of vascular networks of structures located at the same transverse plane of the midbrain. The volume density of the vascular network of the principal oculomotor nucleus is $0.03 \mathrm{~mm}^{0}$, the surface density is $8.35 \mathrm{~mm}^{-1}$, and the length density is $359 \mathrm{~mm}^{-2}{ }^{15}$ The volume density of the blood vessels of the magnocellular part of the nucleus ruber is $0.014 \mathrm{~mm}^{0}$, the surface density is $4.19 \mathrm{~mm}^{-1}$, while the length density is 90.82 $\mathrm{mm}^{-2} .^{16}$ All examined parameters of the vascular network of the SC were lower than the vascular density of the principal oculomotor nucleus and higher than the magnocellular part of the nucleus ruber. Based on these values, it is noticed that the blood vessels of the principal oculomotor nucleus are larger and more convoluted in comparison with the blood vessels of the SC and the magnocellular part of the nucleus ruber, which predisposes the principal oculomotor nucleus to a more frequent vascular occlusion. ${ }^{17,}{ }^{18}$ With ageing, no changes in the studied stereological parameters of the blood vessels of the principal oculomotor nucleus and the magnocellular part of the nucleus ruber were observed, ${ }^{19,16}$ while the blood vessels of 
the SC show a decrease in the length density with age, which means that their tortuosity decreases, which was not seen in other structures at the same transversal level.

Due to the neural connections of the SC and substantia nigra, vascular networks of these two structures were compared. The volume density

\section{Conclusion}

Compared to other structures at the same transversal section through the middle of the SC, the vascular network of SC has an average density. With ageing, all parameters of the vascular network decrease, but length density changes are the only ones that reach statistical significance, which indicates that blood vessels with ageing become less tortuous.

\section{References}

1. Gandhi N, Tsehmaister-Abitbol V, Glikstein R, Torres C. Too much on your "plate"? Spectrum of pathologies involving the tectal plate. Can Assoc Radiol J 2020 May;71(2):186-94.

2. Farrow K, Isa T, Luksch H, Yonehara K. Editorial: The superior colliculus/tectum: cell types, circuits, computations, behaviors. Front Neural Circuits 2019;13:39. doi: 10.3389/fncir.2019.00039.

3. Basso MA, May PJ. Circuits for action and cognition: a view from the superior colliculus. Annu Rev Vis Sci 2017;3:197-226.

4. Bolzani W, Rognone F, Savoldi F, Montalbetti L. Hémorragies du colliculus [Colliculus hemorrhage]. Rev Neurol (Paris) 1996;152(8-9):548-51. French.

5. Duvernoy HM. Human brain stem vessels. 2nd ed. New York: Springer; 2003. p. 47,50,52-55,148.

6. Párraga RG, Ribas GC, Andrade SE, de Oliveira E. Microsurgical anatomy of the posterior cerebral artery in three-dimensional images. World Neurosurg 2011;75(2):233-57.

7. Marinković S, Milisavljević M, Antunović V. [Arteries of brain and spinal cord]. Beograd: Bit inženjering; 2001. p. 76, 77, 96 ,97. Serbian.

8. Smith MS, Laguna JF. Upward gaze paralysis following unilateral pretectal infarction. Computerized tomography correlation. Arch Neurol 1981;38(2):127-9.

9. Tsivgoulis G, Spengos K, Vassilopoulou S, Zakopoulos $\mathrm{N}$, Zis V. Isolated dorsal midbrain infarct: an uncommon cause of pure sensory stroke. Cerebrovasc Dis 2006;21(1-2):139-40.

10. Haines DE. Neuroanatomy in clinical context: an atlas of structures, sections, systems, and syndromes. 9th ed. Philadelphia (PA), USA: Wolters Kluwer Health; 2015.

11. Russ JC, Dehoff RT. Practical stereology. 2nd ed. New York: Kluwer Academic/Plenum Publishers; 2000. of substantia nigra blood vessels was $0.058 \mathrm{~mm}^{0}$, and the surface density was $7.853 \mathrm{~mm}^{-1}$, ${ }^{21}$ which shows that the vascular network of the substantia nigra is much denser than the network of the SC. Due to the higher density of blood vessels, vascular lesions are more common in the anteromedial and anterolateral zone of the mesencephalon, to which the substantia nigra belongs. ${ }^{22}$

\section{Conflict of interest}

None.

\section{Acknowledgements}

None.

12. Kališnik M. [Basis of stereology]. Acta Stereol 1985;4(1):1-148. Serbian.

13. Caplan LR. 'Top of the basilar' syndrome. Neurology 1980;31:152-5.

14. Ortiz de Mendivil A, Alcalá-Galiano A, Ochoa M, Salvador E, Millán JM. Brainstem stroke: anatomy, clinical and radiological findings. Semin Ultrasound CT MR 2013;34(2):131-41.

15. Sladojević I, Krivokuća Z, Bućma T, Gajanin V. [Quantitative analysis of vascular network of oculogyric nerve nuclei]. Med Pregl 2011;64(3-4):143-47. Serbian.

16. Gajanin V, Krivokuća Z, Sladojević I, Bućma T, Šarović Vukajković M. [Quantitative analysis of the magnocellular part of nucleus ruber]. Biomed Istraž 2015;6(2):83-9. Serbian.

17. Ogawa K, Suzuki Y, Oishi M, Kamei S. Clinical study of twenty-one patients with pure midbrain infarction. Eur Neurol 2012;67(2):81-9.

18. Zhang Y, Wang L, He M. Isolated INO as a presentation of midbrain paramedian area lacunar infarction in patients with diabetes. J Clin Neurosci 2017;45:261-4.

19. Sladojević I. [Morphological and morphometric characteristics of extraocular motoneurons]. [MSc thesis]. Banja Luka: Faculty of Medicine, University of Banja Luka; 2009.

20. Comoli E, Coizet V, Boyes J, Bolam JP, Canteras NS, Quirk $\mathrm{RH}$, et al. A direct projection from superior colliculus to substantia nigra for detecting salient visual events. Nat Neurosci 2003;6(9):974-80.

21. Macanović G. [Morphometric analysis of the human substantia nigra]. [MSc thesis]. Banja Luka: Faculty of Medicine, University of Banja Luka; 2012.

22. Kumral E, Bayulkem G, Akyol A, Yunten N, Sirin H, Sagduyu A. Mesencephalic and associated posterior circulation infarcts. Stroke 2002;33(9):2224-31. 